\title{
Phosphotungstic Acid Supported on Magnetic Nanoparticle Used As Selective and Reusable Photocatalyst for Aerobic Oxidation of Benzylic Alcohols
}

\author{
MOZHGAN AFSHARI $^{1 *}$, MARYAM GORJIZADEH ${ }^{1}$ and GHAZAL AFSHAR ${ }^{2}$ \\ 'Department of Chemistry, Shoushtar Branch, Islamic Azad University, Shoushtar, Iran. \\ ${ }^{2}$ Department of Chemistry, Science and Research Branch, Islamic Azad University, Khouzestan, Iran. \\ http://dx.doi.org/10.13005/ojc/290457
}

(Received: November 04, 2013; Accepted: December 10, 2013)

\begin{abstract}
An efficient and selective photochemical method for the oxidation of benzylic alcohols has been achieved using phosphotungstic acid supported on imidazole functionalized silica coated cobalt ferrite nanoparticles as a magnetically photocatalyst in acetonitrile with $\mathrm{O}_{2}$. The photocatalyst has been reused several times, without observable loss of activity and selectivity. Results showed this heterogeneous photocatalyst is more active than the unsupported $\mathrm{H}_{3} \mathrm{PW}_{12} \mathrm{O}_{40}$
\end{abstract}

Key words: Cobalt ferrite, Oxidation, Magnetic nanoparticles, Surface functionalization, Phosphotungstic acid.

\section{INTRODUCTION}

The oxidation of alcohols into their corresponding carbonyl compounds represents a fundamentally important functional group transformation and occupies a prominent position in modern synthetic organic chemistry ${ }^{1}$. Many thermal catalytic systems have been designed and developed for the oxidation of alcohols ${ }^{2-13}$. Most of these systems are particularly effective for activated (benzylic and allylic) alcohols. However, the search for suitable green catalysts is still a significant challenge. In this context, photoreactions are promising processes and the development of photocatalysts is a subject that is now receiving much attention. An important class of photocatalysts that has received more attention is the polyoxometalates (POMs) ${ }^{14}$.
Despite of some advantages, owing to the good solubility of $\mathrm{POMs}$ in polar solvents heterogenization of the homogeneous catalysts is very attractive to overcome the difficulty of recovery. A possible method is immobilizing POMs species in the surface of magnetic particles which can be separated and recovered from the reaction system by applying an appropriate magnetic field ${ }^{15-25}$. Moreover compared to conventional supported POMs photocatalysts, the nanometer sizes of the magnetic photocatalyst provide a larger surface area, which subsequently enables higher efficiency in photo-catalytic reactions. However, to the best of our knowledge, only a few reports have appeared in the literature about photocatalytic activity of polyoxometalates supported on MNPs. The first example of immobilization of POMs on MNPs, as efficient photocatalysts for 
degrading Rhodamine B was reported by Zheng et.al ${ }^{26}$; they prepared magnetic photocatalyst by grafting polyoxometalates (POM) anions onto $\mathrm{Fe}_{3} \mathrm{O}_{4}$ nanoparticles via a layer of $\mathrm{Ag}$. In another report, Li et.al fabricates the amino functionalized magnetic core-shell mesoporous microspheres, which play as the support for immobilizing $\mathrm{H}_{3} \mathrm{PW}_{12} \mathrm{O}_{40}$ onto the surface of the microspheres. This composite also shows high efficiency in decomposing Rhodamine B under UV irradiation ${ }^{27}$.

In the present work, the phosphotungstic acid supported on imidazole functionalized silica coated cobalt ferrite nanoparticles (PTA/Si-imid@ Si-MNPs) was used, for the first time, as an efficient and magnetically recyclable heterogeneous photocatalyst for aerobic oxidation of various primary and secondary benzylic alcohols into the corresponding carbonyl compounds.

\section{EXPERIMENTAL}

\section{General}

All chemicals were purchased from Sigma-Aldrich or Merck and used as received. Phosphotungstic acid supported on magnetic nanoparticles was prepared using the procedure reported by Kooti et al. ${ }^{18}$. FT-IR spectra were obtained using BOMEM MB-Series 1998 FT-IR spectrometer. Magnetic properties of all nanoparticles were measured with a vibrating sample magnetometer (VSM, Meghnatis Daghigh Kavir Coumpany) at room temperature. NMR spectra were recorded in $\mathrm{CDCl}_{3}$ on a Bruker Advanced DPX $400 \mathrm{MHz}$ spectrometer.

\section{Catalytic studies}

In a Pyrex flask equipped with a magnet bar, a solution of the alcohol ( $5 \mathrm{mmol})$ in acetonitrile $(10 \mathrm{~mL})$ was prepared. To this solution PTA/Si-imid@ Si-MNPs (50 mg) was added and purged with $\mathrm{O}_{2}$ and joined through an inlet tube to a balloon filled with $\mathrm{O}_{2}$ before irradiation.

The suspension was vigorously stirred and irradiated with a high pressure $400 \mathrm{~W}$ mercury lamp using a cut-off Pyrex filter at room temperature. The progress of the reaction was monitored by TLC. At the end of the reaction the organic layer was simply decanted by means of an external magnet. The decanted solution was purified on a silica-gel plate or a silica-gel column to obtain the pure product. The identities of the products were confirmed by FT-IR and ${ }^{1} \mathrm{H}$ NMR spectral data.

\section{RESULTS AND DISCUSION}

\section{Characterization OF PTA/Si-imid@ Si-MNPs}

The magnetic core was analyzed by XRD and the peaks are compatible with pure $\mathrm{CoFe}_{2} \mathrm{O}_{4}$ phase (JCPDS PDF \#221086), indicating the retention of cubic reverse spinel structure of $\mathrm{CoFe}_{2} \mathrm{O}_{4}$ during coating and functionalization (Fig. 1).

The magnetic properties of the silica coated cobalt ferrite (Si-MNPs) and PTA/Si-imid @ Si-MNPs were summarized in Table 1. The decrease in mass saturation magnetization may be ascribed to the contribution of the non-magnetic silica shell and functionalized groups

The presence PTA was confirmed by IR (see Fig. 2). The TEM micrograph of the nanoparticles is shown in Fig.3. TEM image indicated that most of the prepared nanoparticles are spherical shaped and have size less than 20-30 $\mathrm{nm}$. Moreover, the tungsten content of the catalyst, as determined by ICP-AES, was $0.98 \mathrm{mmol} / \mathrm{g}$

\section{Alcohols}

In order to optimize the reaction conditions for the oxidation of benzylic alcohols various experiments were carried out at room temperature using 1-phenylethanol in the presence of PTA/Siimid@ Si-MNPs as photocatalyst under $\mathrm{O}_{2}$.

To optimize the catalyst requirement, the amount of catalyst was changed between 20 and 150

Table 1. Magnetic property of the as-fabricated nanoparticles

\begin{tabular}{lccc}
\hline compound & $\begin{array}{c}\mathbf{H}_{\mathbf{c}} \\
\text { (Oe) }\end{array}$ & $\begin{array}{c}\mathbf{M}_{\mathbf{r}} \\
\text { (emu/g) }\end{array}$ & $\begin{array}{c}\text { Ms } \\
\text { (emu/g) }\end{array}$ \\
\hline MNPs & 796.1 & 24.63 & 59.49 \\
Si-MNPs & 861.05 & 16.40 & 38.93 \\
PTA/Si-imid @ & & & \\
Si-MNPs & 688.05 & 6.54 & 21.84 \\
\hline
\end{tabular}


Table 2. Oxidation of alkenes PTA/Si-imid@ Si-MNPs photocatalyst a

\begin{tabular}{|c|c|c|c|c|}
\hline Entry & Alochol & Produt & Time (h) & Yield $^{\mathrm{b}} \%$ \\
\hline 1 & & & 1.5 & 82 \\
\hline 2 & & & 1 & 92 \\
\hline 3 & & & 1 & 94 \\
\hline 4 & & & 1 & 92 \\
\hline 5 & & & 1.5 & 81 \\
\hline 6 & & & 1 & 92 \\
\hline 7 & & & 0.5 & 91 \\
\hline 8 & & & 3.5 & 65 \\
\hline 9 & & & 1.5 & 66 \\
\hline 10 & & & 1 & 72 \\
\hline 11 & & & 1 & 90 \\
\hline 12 & & & 1 & 94 \\
\hline 13 & & & 15 & 75 \\
\hline 14 & & & 1.5 & 78 \\
\hline 15 & & & 1.5 & 85 \\
\hline $16^{c}$ & & & 1.5 & 86 \\
\hline
\end{tabular}

aReaction conditions: allcohol(5mmol), catalyst $(50 \mathrm{mg})$, acetonitrile $(10 \mathrm{ml}), \mathrm{O}_{2}$ atmosphere, rt. blsolated yields, ccatalyst : tenth recycled catalyst 


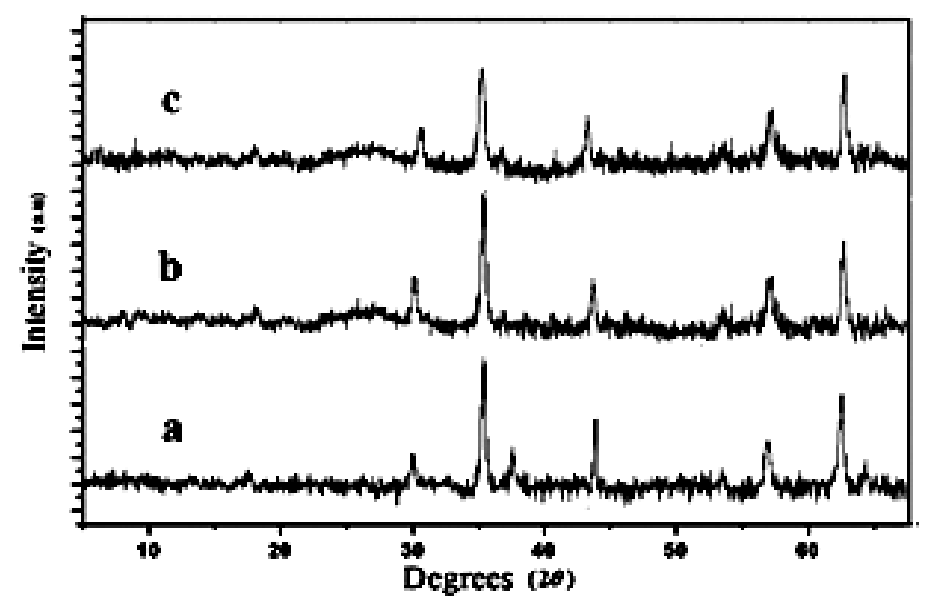

Fig. 1: XRD pattern of (a) MNPs, (b) Si-MNPs and (c) PTA/Si-imid@ Si-MNPs

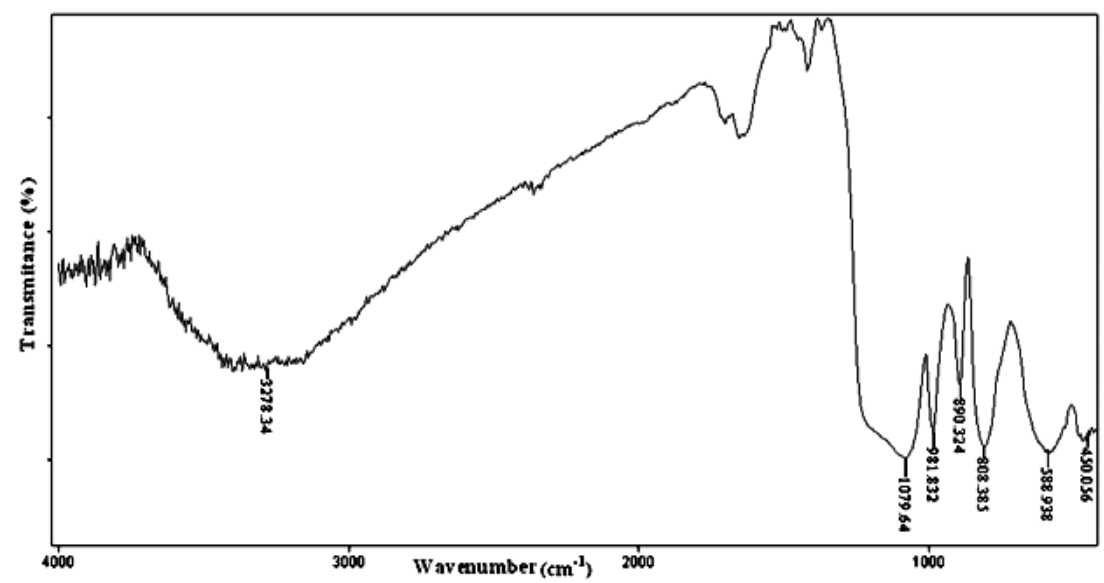

Fig. 2: FT-IR spectrum of PTA/Si-imid@ Si-MNPs

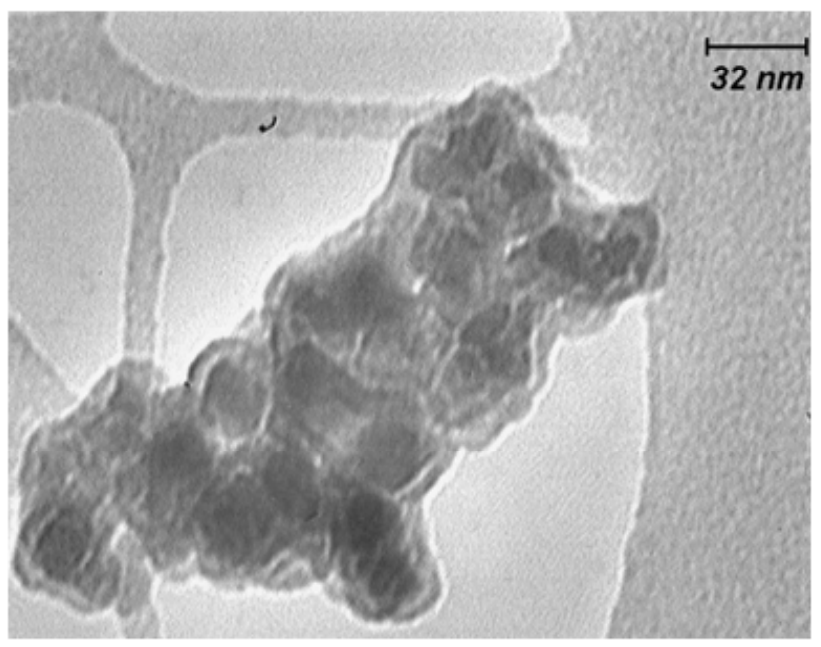

Fig. 3: TEM image of PTA/Si-imid@ Si-MNPs 


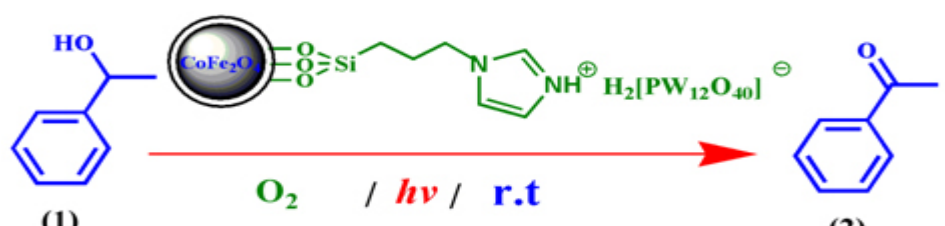

(1)

Scheme 1: Oxidation of 1-phenylethanol using PTA/Si-imid@ Si-MNPs catalyst

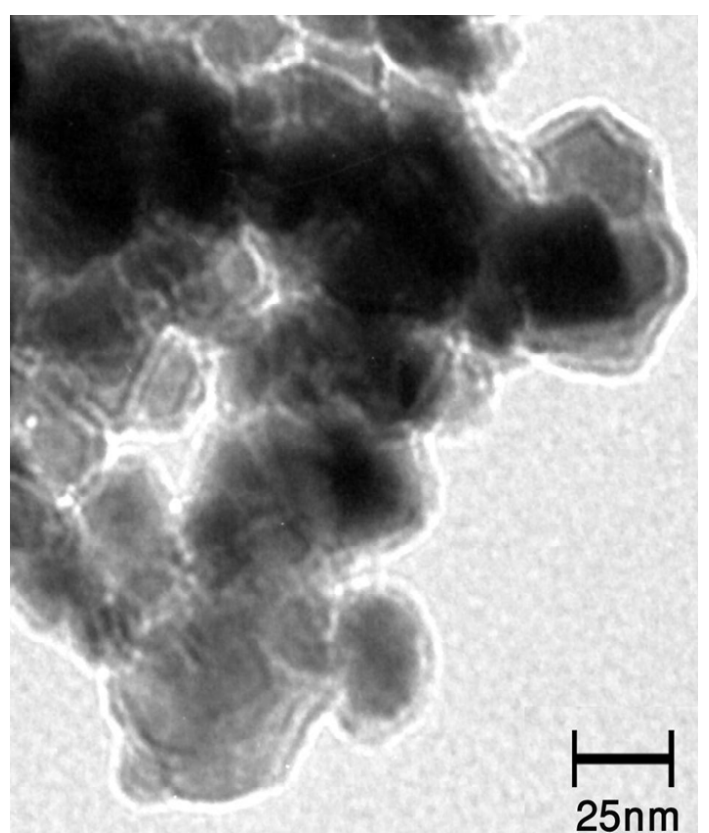

Fig. 4: TEM image of recycled PTA/Si-imid@ Si-MNPs

$\mathrm{mg}$ per $5 \mathrm{mmol}$ 1-phenylethanol alcohol. Increase in the yield of acetophenone was observed when the amount of catalyst was increased from 20 to 50 $\mathrm{mg}$ but the yield remained almost same with further increment of catalyst amount up to $150 \mathrm{mg}$. An optimum of $50 \mathrm{mg}$ catalyst in the reaction mixture is ideal for achieving the best yield. We have also accomplished the oxidation of 1-phenylethanol in the different solvents such as n-hexane, acetone, toluene, cyclohexan, chloroform, dichloromethane and also acetonitrile under irradiation at room temperature. Among the solvents examined, we found acetonitrile to be the best for this protocol probably because of its photoinertness and all the alcoholic substrates and their products were soluble in this solvent.

For investigating the chemoselectivity, a mixture of 1-phenylethanol and 2-phenylethanol was subjected to $1 \mathrm{~h}$ irradiation in the presence of PTA/Siimid@ Si-MNPs under $\mathrm{O}_{2}$. Result indicated that the former was oxidized to acetophenone in $92 \%$ yield while 2-phenylethanol was recovered completely.

Moreover, we have also examined the photocatalytic oxidation of 2-phenylethanol under pure $\mathrm{O}_{2}$ (1 atm), air and $\mathrm{N}_{2}$. It was found that the efficiency for the photocatalytic oxidation of 2-phenylethanol depends on the concentration of $\mathrm{O}_{2}$, the photocatalytic activity was higher in the presence of pure $\mathrm{O}_{2}$ than in air, and almost no activity was observed under $\mathrm{N}_{2}$.

At optimum conditions we observed that when a heterogeneous mixture of this alcohol and PTA/Si-imid@Si-MNPs was irradiated under $\mathrm{O}_{2}$ atmosphere in acetonitrile, acetophenone (2) was formed as the only product in $92 \%$ yield within $1 \mathrm{~h}$ at room temperature (see scheme1).

On the other hand, 1 was oxidized to acetophenone in a $72 \%$ yield within $2.5 \mathrm{~h}$ when pure $\mathrm{H}_{3} \mathrm{PW}_{12} \mathrm{O}_{40}$ (PTA) was used as the photocatalyst under the same reaction conditions. This result clearly indicated that immobilized $\mathrm{H}_{3} \mathrm{PW}_{12} \mathrm{O}_{40}$ on magnetic nanoparticles is more active than the bulk PTA and PTA/SiO .

The obtained preliminary results for the oxidation of 1-phenylethanol were encouraging and to extend the scope of this heterogeneous catalytic system, the oxidation of further alcohols was carried out under the optimized conditions. The given results in Table 2 show that this procedure was clean and all the alcohols were converted into the corresponding carbonyl compounds in moderate to excellent yields without over-oxidation to carboxylic acids.

Furthermore, this reaction is highly selective for vicinal diols in oxidizing only the secondary hydroxy group \pm to the benzene ring (Table 2 , entries 15 ). 
Therefore, it is a method of choice for the oxidation of benzylic $\mathrm{OH}$ in the presence of non-benzylic $\mathrm{OH}$. On the basis of previously reported mechanisms, PTA in PTA/Si-imid@ Si-MNPs nanocomposite is activated by light absorption and reacts with alcohol to give the corresponding carbonyl compound. In the presence of dissolved dioxygen in suspension, the thermal oxidation of the reduced species of PTA takes place at room temperature.

Easy and rapid separation of the catalyst by magnetism is the most advantageous feature of this catalyst. The recovery and reusability of the catalyst was studied using 1-phenylethanol as model substrate. Catalyst recycling experiments were achieved by fixing the catalyst magnetically at the bottom of the flask and the solution was decanted after each run. The left solid was washed with acetonitrile twice, and fresh substrate dissolved in acetonitrile is introduced into the flask, allowing the system to proceed for next run. It was found that the developed catalyst could be used at least ten times without any noticeable loss of its catalytic activity (Table 2, entries 16). ICP-AES analysis has shown that leaching of the catalyst from support $(0.8 \% \mathrm{~W})$ occurred only in the first run and no leaching was observed in the next runs. Furthermore, the FT-IR, XRD and TEM (see Fig. 4) of the recovered catalyst showed no change after using the catalyst for ten times.
Compared with the other heterogeneous POMs catalysts ${ }^{28-30}$, our catalyst (PTA/Si-imid @ Si-MNPs) has shown much more superiority. The attractive features of this method are high yield, short reaction time, clean reaction profiles, simple work-up procedure, ease of separation, and recyclability of the magnetic catalyst, as well as the ability to tolerate a wide variety of substitutions in the reagents.

\section{CONCLUSION}

In conclusion, a novel and efficient procedure for the oxidation of primary and secondary benzylic alcoholshas been developed using immobilization of Phosphotungstic acid (PTA) on imidazole-functionalized silica coated cobalt ferrite nanoparticles as a green and recyclable photocatalyst, in the presence of $\mathrm{O}_{2}$ as the sole catalyst reoxidant. Moreover, the immobilized PTA catalyst could be easily recovered by simple magnetic decantation and reused at least five times without significant loss of activity.

\section{ACKNOWLDEGMENTS}

The authors wish to acknowledge the support of this work by the Research Council of Shoushtar Branch, Islamic Azad University, Shoushtar, Iran

\section{REFERENCES}

1. R.A. Sheldon, I. W. C. E Arends, G.-J.Ten Brink, A .Dijksman, Acc. Chem. Res. 35, 774 (2002).

2. P.Chaudhuri, M. Hess, J. Muller, K. Hildenbrand, E. Bill, T. Weyhermuller, K. Wieghardt, J. Am. Chem. Soc. 121,9599 (1999).

3. I.E. Marko, P.R Giles, M. Tsukazaki, I. ChelleRegnaut, A.Gautier, S.M. Brown, C.J. Urch, J. Org. Chem.64, 2433 (1999).

4. A. Haimov, R. Neumann, R. Chem. Commun. 876 (2002).

5. Steinhoff, B. A.; Fix, S. R.; Stahl, S. S. J. Am. Chem. Soc. 124, 766 (2002).

6. G. Ragagnin, B. Betmezeier, S.Quici, P. Knochel, Tetrahedron, 58, 3985 (2002).

7. G. Csjernyik, A.H Ell, L. Fadini, B. Pugin, J.E
Backvall, J. Org.Chem. 67,1657 (2002).

8. J. Muldoon, S.N. Brown, Org. Lett. 4, 1043 (2002).

9. B.Z. Zhan, M.A. White, T.K. Sham, J.A. Pincock, R.J. Doucet, K.V.R. Rao, K.N. Robertson, T.S. Cameron, J. Am. Chem. Soc.125, 2195 (2003).

10. Mallat, T.; Baiker, A. Chem. Rev. 104, 3037 (2004).

11. Lee, M.; Chang, S. Tetrahedron Lett. , 41, 7507 (2000)

12. Lorber, C. Y.; Smidt, S. P.; Osborn, J. A. Eur. J. Inorg.Chem. 655(2000).

13. Ciriminna, R.; Pagliaro, M. Chem. Eur. J. 9, 5067(2003).

14. E. Papaconstantinou, J. Chem. Soc., Chem. 
Commun. 12 (1982).

15. Z. Zhang, F. Zhang, Q. Zhu, W. Zhao, B. Ma, Y. Ding, J. Colloid Interface Sci. 360,189 (2011).

16 X. Cui, D. Yao, H. Li, J. Yang, D. Hu, J. Hazard. Mater. 205, 17 (2012).

17 X. Zheng, L. Zhang, J. Li, S. Luo, J.P. Cheng, Chem. Commun. 47, 12325 (2011).

18 M. Kooti, M. Afshari, Mater. Res. Bull. 47, 3473 (2012).

19 M. Bagheri, M.Masteri-Farahani b, n, M.Ghorbani, J. Magn. Magn. Mater. 327, 58 (2013).

20. E. Rafiee, S. Eavani J. Mol. Catal.A: Chem., 373,30 (2013)

21. Y. Chen, F. Zhang , Y. Fang, X. Zhu, W. Zhen, R. Wang, J. Ma, Catal Commun.,38, 54 (2013).

22. A. Kong, P. Wang, H. Zhang, F. Yang, S.P. Huang, Y. Shan, Appl Catal. A: Gen 417-418,
183 (2012).

23. M. Masteri-Farahania, , J. Movassaghb, F. Taghavib, P. Eghbalib, F. Salimib Chem. Engin. J., 184: 342 (2012).

24. S. Neelakumari and G. Raveendran, Orient. J. Chem., 29(1): 341-346 (2013).

25. N. Montazeri, S. F. Ayoubi, K. Pourshamsian and F. Bashtini, Orient, J. Chem., 28(1): 303307 (2012).

26. W. Qiu, Y. Zheng, K. A. Haralampides, Chem. Engin. J., 125: 165 (2007).

27. L. Zhao, Y. Chi, Q. Yuan, N.Li, W. Yan, X.Li, J. Colloid Interface Sci. 390: 70 (2013).

28. S.Farhadi, M. Zaidi, Appl Cata. A: Gen, 354: 119 (2009).

29. S. Farhadi, A. Zabardasti, Z. Babazadeh, Tetrahedron Lett., 47: 8953 (2006).

30. S. Farhadi, M. Afshari, M.Maleki, Z.Babazadeh, Tetrahedron Lett., 46: 8483 (2005). 